\title{
IDENTIFICAÇÃO DO PERFIL ANTROPOMÉTRICO DE INDIVÍDUOS ATENDIDOS EM UM EVENTO DE QUALIDADE DE VIDA
}

\section{IDENTIFICATION OF THE ANTROPOMETRIC PROFILE OF INDIVIDUALS ATTENDING A QUALITY OF LIFE EVENT}

\author{
Edeli Simioni de Abreu ${ }^{1}$ \\ Rosana Farah Simony ${ }^{2}$ \\ Débora Helena Silveira Dias ${ }^{3}$ \\ Fernanda Heitzmann Martins ${ }^{3}$ \\ Flávia Regina Oliveira Ribeiro ${ }^{3}$
}

RESUMO: É notório o aumento da prevalência de sobrepeso e obesidade na população brasileira. Estudos recentes indicam uma projeção de comportamento epidêmico. Este estudo descreveu o perfil nutricional de indivíduos atendidos em um evento de Qualidade de Vida, realizado em uma Universidade localizada no município de São Paulo. Trata-se de um estudo transversal, descritivo e com coleta de dados primários, com amostra de 443 indivíduos de ambos os gêneros, realizado em uma Universidade do município de São Paulo, no dia 30 de abril de 2010. O evento fez parte de um programa de qualidade de vida, já instituído na Universidade. Foram coletados os dados antropométricos (peso, altura e circunferência de cintura), e avaliou-se o estado nutricional por meio do índice de massa corporal e índice de conicidade. Participaram do evento 443 indivíduos, sendo $48 \%$ ( $n=213$ ) do sexo feminino e $52 \%$ ( $n=230$ ) do sexo masculino, com média de idade $28,7( \pm 3,9)$ anos. $A$ amostra estudada apresentou uma prevalência de 26,9\% de sobrepeso e 12,6\% para obesidade, maior nos homens quando comparados às mulheres. Em relação ao risco de doenças cardiovasculares, segundo a circunferência de cintura e índice de conicidade, a maior parte (67,7\%) não apresentou risco, porém esses valores foram maiores entre as mulheres. O estudo permitiu concluir que quase $40 \%$ da população estava com excesso de peso. Dessa maneira, conclui-se que é essencial a realização de programas de intervenção nutricional para detectar possíveis riscos, e promover melhorias no estado de saúde dos indivíduos, para lhes assegurar uma maior qualidade de vida.

Palavras-chave: antropometria; qualidade de vida; índice de conicidade; transição nutricional.

ABSTRACT: Overweight and obesity is known to be increasing in prevalence in the Brazilian population. Recent studies indicate a projection of epidemic behavior. This study described the nutritional profile of individuals attending a quality of life event at a University located in the city of São Paulo. This cross-sectional descriptive study used primary data collection with a sample of 443 individuals of both genders, conducted at a University in São Paulo, on April 30, 2010. The event was part of a program about quality of life already established at the University. Anthropometric data were collected (weight, height, and waist circumference), and the nutritional state was evaluated using the body mass index, and conicity index. In attendance were 443 individuals including 48\% ( $n=213)$ females and 52\% ( $n=230)$ males, with average age of 28.7 (3.9 \pm ) years. The sample studied presented a $26.9 \%$ prevalence of overweight and $12.6 \%$ to obesity, which was higher in men when compared with women. In relation to the risk of cardiovascular disease according to the circumference of the waist and conicity index, most (67.7\%) did not run the risk, but these rates seem to be higher among women. The study found that almost $40 \%$ of the population was overweight. Therefore, it was concluded that nutrition intervention programs are essential to detect possible risks and to promote improvements in individual's health status to ensure a better quality of life.

Keywords: anthropometry; quality of life; conicity index; nutritional transition.

\footnotetext{
1 Doutora em Saúde Pública - Universidade de São Paulo - USP e Professora Adjunta I da Universidade Presbiteriana Mackenzie - UPM. E-mail: edelisabreu@gmail.com.

${ }^{2}$ Doutora em Medicina - Universidade Federal de São Paulo e Professora Adjunta I da UPM. E-mail: rfarah@mackenzie.br.

3 Graduada em Nutrição - UPM. E-mails: deborahsd.88@gmail.com; fernandaheitzmann@hotmail.com; flavinhacavaco@uol.com.br.
} 


\section{INTRODUÇÃO}

O estado nutricional de um indivíduo expressa se o seu grau de necessidades fisiológicas, obtido por nutrientes, está sendo alcançado para manter a composição e homeostasia do organismo, resultando do equilíbrio entre ingestão e necessidade de nutrientes. Suas alterações contribuem para - aumento da morbimortalidade, que compreendem estados de desnutrição, sobrepeso e obesidade (ACUÑA; CRUZ, 2004).

A desnutrição predispõe a uma série de complicações graves, incluindo tendência a infecções, carências nutricionais, falência respiratória, cardíaca, renal e hepática. Por outro lado, o sobrepeso e a obesidade também são fatores de risco para agravos à saúde, sendo que entre os mais frequentes estão doença isquêmica do coração, hipertensão arterial, acidente vascular cerebral, diabetes mellitus tipo 2 e problemas psicológicos (LOLIO; LATORRE,1991; ACUÑA; CRUZ, 2004).

Ao mesmo tempo em que declina a ocorrência da desnutrição em crianças e adultos, aumenta a prevalência de sobrepeso e obesidade na população brasileira. Os resultados dos estudos nos últimos 30 anos indicam uma projeção de comportamento epidêmico do problema. Percebe-se um antagonismo de tendências temporais entre desnutrição e obesidade, definindo uma das características marcantes do processo de transição nutricional do país (BATISTA FILHO; RISSIN, 2003).

As tendências dessa transição nutricional, ocorrida nestes últimos anos, convergem para uma dieta mais rica em gorduras, açúcares e alimentos refinados, reduzida em carboidratos complexos e fibras, além do declínio progressivo da atividade física. Esses fatores são contribuintes para alterações da composição corporal, principalmente do aumento da gordura abdominal (FRANCISCHI et al., 2000).

O acúmulo de gordura abdominal vem sendo descrito como o tipo de obesidade que oferece maior risco à saúde dos indivíduos. A incidência de algumas doenças, como o aumento do risco de infarto do miocárdio, acidente vascular cerebral e morte prematura, incidência de diabetes, aterosclerose, cálculo urinário, é elevada em pessoas com obesidade visceral (PITANGA; LESSA, 2005).

A Organização Mundial de Saúde (OMS) indica a antropometria como método mais útil para identificar alterações do estado nutricional. O método mais utilizado é o Índice de Massa Corpórea (IMC), tendo como equação peso (em $\mathrm{kg}$ ) dividido pelo quadrado da altura (em metros). É um método de fácil aplicação, possui baixo custo, não é invasivo, é universalmente aplicável e tem boa aceitação da população (ABRANTES; LAMOUNIER; COLONISMO, 2003; SAMPAIO; FIGUEIREDO, 2005).

Estudos realizados com amostras representativas de indivíduos adultos de ambos os sexos, demonstraram que o IMC correlaciona-se bem com indicadores antropométricos de gordura abdominal ou visceral (circunferência da cintura), além de ter relação direta com a massa de gordura corporal total (RANKINEN et al., 1999; LEITE et al., 2002).

Nesse contexto, justifica-se a realização do presente estudo, considerando a mudança de perfil epidemiológico da população brasileira e a possibilidade de prevenção das doenças crônicas não 
transmissíveis, diagnosticando o perfil antropométrico, em indivíduos adultos.

O estudo teve como objetivo descrever - perfil antropométrico de indivíduos atendidos em um evento de qualidade de vida, realizado em uma Universidade localizada no município de São Paulo.

\section{METODOLOGIA}

Trata-se de um estudo transversal, descritivo e com coleta de dados primários. A amostra foi constituída por 443 indivíduos de ambos os gêneros, com idade igual ou superior a 20 anos, num evento realizado em uma Universidade localizada no município de São Paulo, no dia 30 de abril de 2010 , das $9 \mathrm{~h}$ às $18 \mathrm{~h}$. O evento fez parte de um programa de qualidade de vida, já instituído na Universidade, sendo que os dados foram coletados por alunos de graduação do curso de nutrição, treinados.

Em um primeiro momento, foram aferidas as medidas antropométricas de peso $(\mathrm{kg})$ e altura $(\mathrm{m})$. Para a coleta de peso, foi utilizada uma balança digital calibrada, da marca Toledo $\AA$ (capacidade de $150 \mathrm{~kg}$ e variação de $0,1 \mathrm{~kg}$ ), com antropômetro acoplado (com precisão de 0,1 $\mathrm{cm}$ ), o qual foi utilizado para registro da altura. Todos os participantes foram medidos eretos, descalços, com os calcanhares juntos, costas retas, braços estendidos ao lado do corpo e cabeça posicionada num ângulo de $90^{\circ} \mathrm{com} 0$ pescoço, seguindo as recomendações do Manual de Técnicas e Procedimentos do Ministério da Saúde. O índice de massa corporal (IMC) foi calculado dividindo-se o peso $(\mathrm{kg})$ pelo quadrado da altura $\left(\mathrm{m}^{2}\right)$ e classificado de acordo com os pontos de corte recomendados pela Organização Mundial da Saúde.

A circunferência da cintura (CC) foi aferida em torno do tronco, dois dedos acima da cicatriz umbilical (indivíduos em pé), com uma fita métrica inelástica (com precisão de $1 \mathrm{~mm}$ ), sem fazer pressão, sendo utilizada a classificação da OMS, para avaliação do risco de complicações metabólicas, considerando-se os pontos de corte para homens, para risco aumentado, os valores de CC entre 94 e $102 \mathrm{~cm}$, e, substancialmente aumentado valores de CC iguais ou superiores a $102 \mathrm{~cm}$; e, para as mulheres, risco aumentado de CC entre $80 \mathrm{e}$ $88 \mathrm{~cm}$, e muito aumentado, para valores iguais ou superiores a $88 \mathrm{~cm}$.

Para avaliação de risco de obesidade central e consequente risco de desenvolvimento de doenças coronarianas, foi utilizado o índice de conicidade, calculado por meio das medidas de peso, estatura e circunferência da cintura, utilizando-se a seguinte equação matemática de Valdez (1991).

$$
\text { Índice } \mathrm{C}=\frac{\text { Circunferência Cintura }(\mathrm{m})}{0,109 \sqrt{\frac{\text { Peso Corporal }(\mathrm{kg})}{\text { Estatura }(\mathrm{m})}}}
$$

Dessa forma, ao ser calculado o índice de conicidade, tem-se a seguinte classificação: para mulheres, o ponto de corte foi de 1,18; e, para homens, 1,25, os valores acima desses pontos de corte foram considerados como alto risco para desenvolvimento de doenças coronarianas (PITANGA; LESSA, 2005).

Após a aferição das medidas antropométricas, os indivíduos receberam orientação sobre alimentação saudável e 
práticas de atividade física, de acordo com os dez passos para uma alimentação saudável, do Ministério da Saúde (BRASIL, 2006).

Por se tratar de uma análise exploratória de dados, estes foram abordados por meio da estatística descritiva simples, cujo objetivo básico foi sintetizar uma série de valores de mesma natureza, utilizando-se médias, desvio padrão, frequências e porcentagens.

Para a realização da analise estatística, foram utilizados os testes estatísticos não paramétricos, pois as variáveis quantitativas não tinham distribuição normal. As diferenças de proporções, em relação à variável sexo, foram analisadas pelo teste do Quiquadrado $\left(\chi^{2}\right)$. As análises foram realizadas com a versão 17.0, do SPSS (SPSS Inc,
Chicago, EUA), e foi considerado o nível de significância de $5 \%$.

\section{RESULTADOS E DISCUSSÃO}

Participaram do evento 443 indivíduos, sendo que $48 \% \quad(n=213)$ eram do sexo feminino e $52 \%(n=230)$ do sexo masculino, com idade igual ou superior a 20 anos, com média de idade de 28,7 $( \pm 11,7)$ anos.

A amostra apresentou um IMC médio de $24,7( \pm 4) \mathrm{kg} / \mathrm{m}^{2}$, sendo que $26,9 \%$ apresentaram sobrepeso e $12,6 \%$ obesidade (Fig. 1) e, aparentemente, isso foi maior nos homens, quando comparados às mulheres (33,4\% vs. $19,7 \%$ para sobrepeso e $14,3 \%$ vs. $10,7 \%$ para obesidade) (Fig. 2).

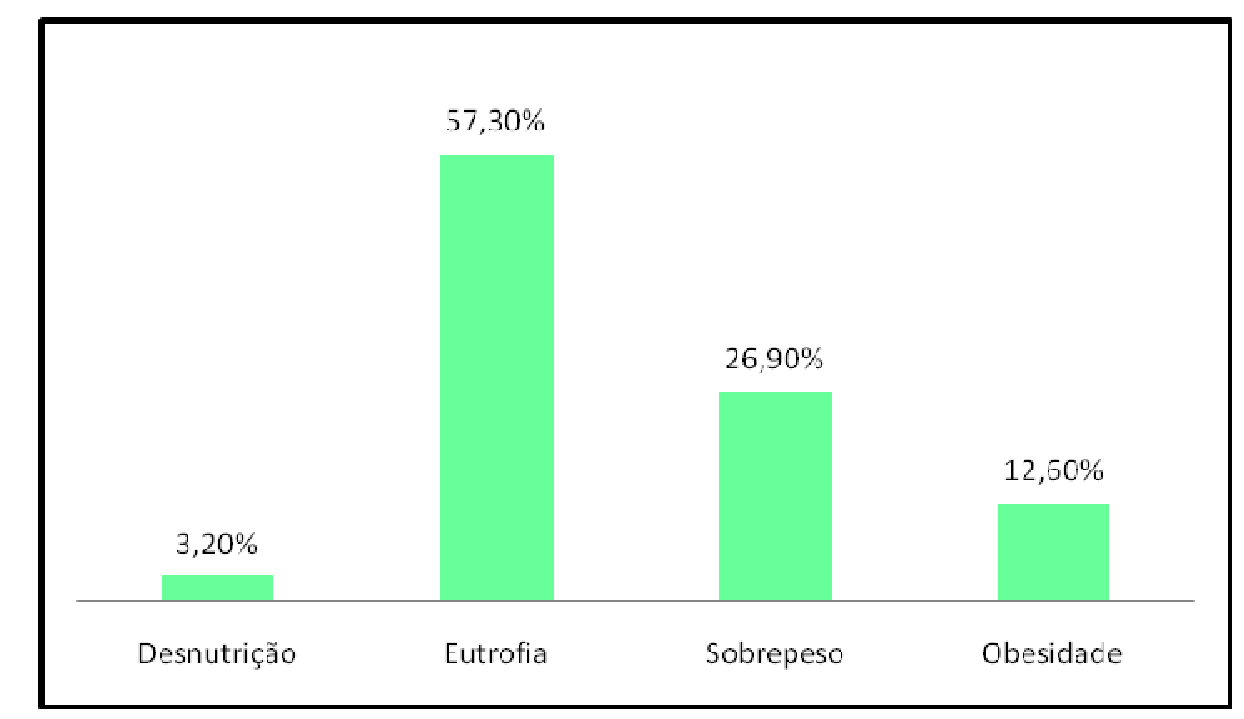

Fig. 1 - Classificação do estado nutricional, segundo IMC, da amostra estudada, São Paulo, 2010. 


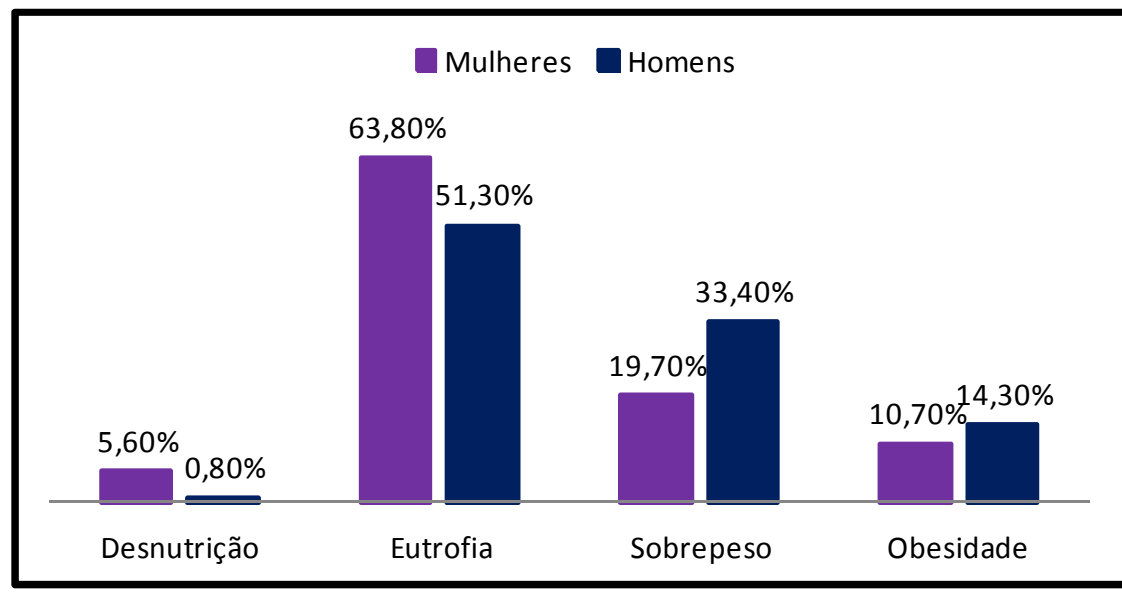

$$
\chi^{2}=19,991 ; p<0,001
$$

Fig. 2 - Classificação do estado nutricional, segundo IMC e sexo, da amostra estudada, São Paulo, 2010.

Os dados deste estudo confirmam o que a maioria dos estudos relata, ou seja, o aumento da prevalência de obesidade na população brasileira.

Trabalhos semelhantes realizados com populações adultas encontraram valores de obesidade (IMC $\geq 30 \mathrm{~kg} / \mathrm{m}^{2}$ ) que variam de $17,8 \%$ (SOUZA et al., 2003) a $38 \%$ (CERVATO et al., 1997).

A Fig. 3 mostra que apesar da maior parte da população $(67,7 \%)$ não apresentar risco para doenças cardiovasculares, conforme a circunferência da cintura, a análise, segundo o sexo, mostra que $18,5 \%$ e $22,3 \%$ das mulheres apresentaram risco aumentado e muito aumentado para doenças cardiovasculares (Fig. 4).

Em relação aos homens, apesar de apresentarem maior risco para sobrepeso e obesidade, pelo IMC, apresentaram baixo risco para doenças cardiovasculares, pela circunferência de cintura.

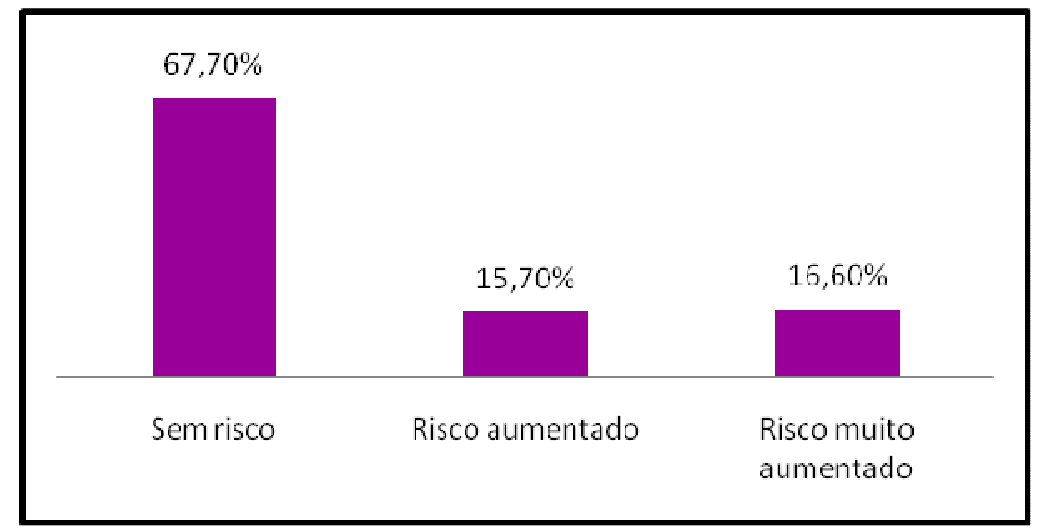

Fig. 3. Distribuição percentual da amostra, segundo risco de desenvolvimento de doenças cardiovasculares, de acordo com a circunferência da cintura (CC), São Paulo, SP, 2010. 


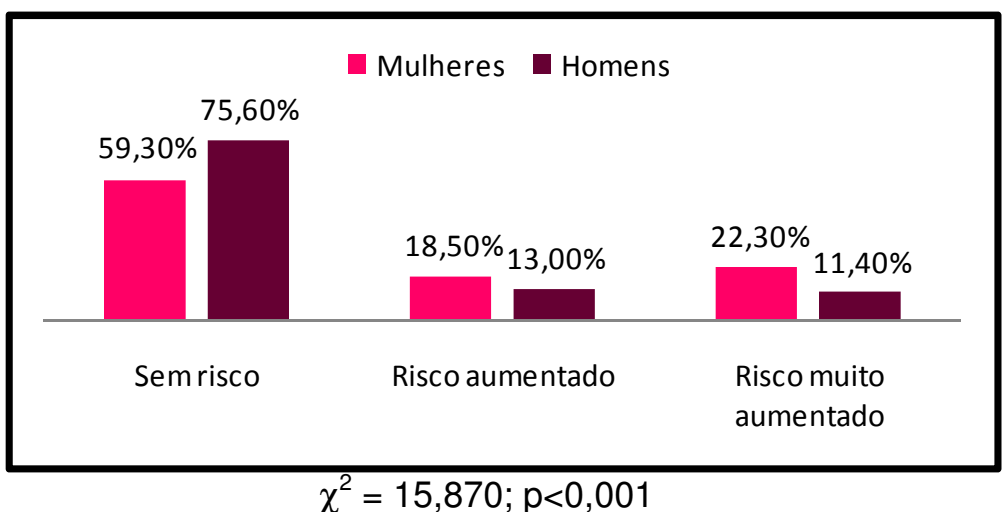

Fig. 4 - Distribuição percentual da amostra, segundo risco de desenvolvimento de doenças cardiovasculares, de acordo com a circunferência da cintura (CC), segundo o sexo, São Paulo, SP, 2010.

Esses resultados são semelhantes aos dados apresentados por Coutinho et al. (1991), os quais confirmam que as mulheres têm maior probabilidade de apresentar acúmulo de gordura abdominal quando comparadas com os homens.
No estudo em questão, pôde-se observar que mesmo $57,3 \%$ da amostra estando em eutrofia, cerca de $10 \%$ destes apresentaram risco aumentado e muito aumentado para doenças cardiovasculares (Fig. 5).

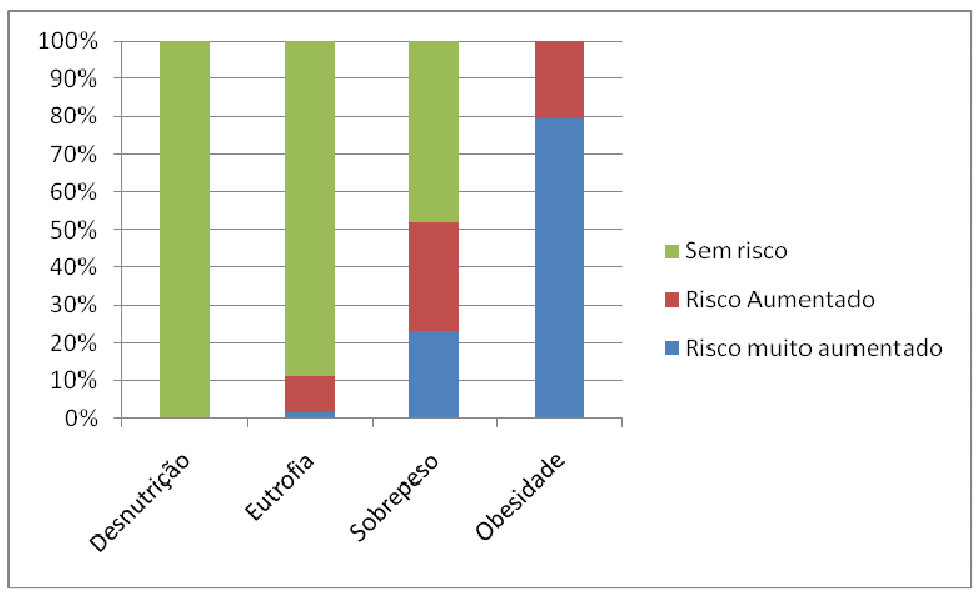

Fig. 5 - Risco de comorbidades de acordo com a classificação do IMC, da circunferência da cintura, para adultos, São Paulo, 2010.

Souza et al. (2003), estudando a prevalência de obesidade e fatores de risco cardiovascular em uma população de adultos no Rio de Janeiro, observaram que mais de $50 \%$ dos emtrevistados foram classificados como portadores de algum risco para eventos cardiovasculares, segundo o IMC e circunferência abdominal.
Em relação ao índice de conicidade (IC), grande parte (63\%) apresentou baixo risco de desenvolvimento de doenças coronarianas (DC) (Fig. 6). O que era de se esperar, já que, como relatado, $67,7 \%$ apresentaram-se sem risco, em relação à circunferência de cintura. 


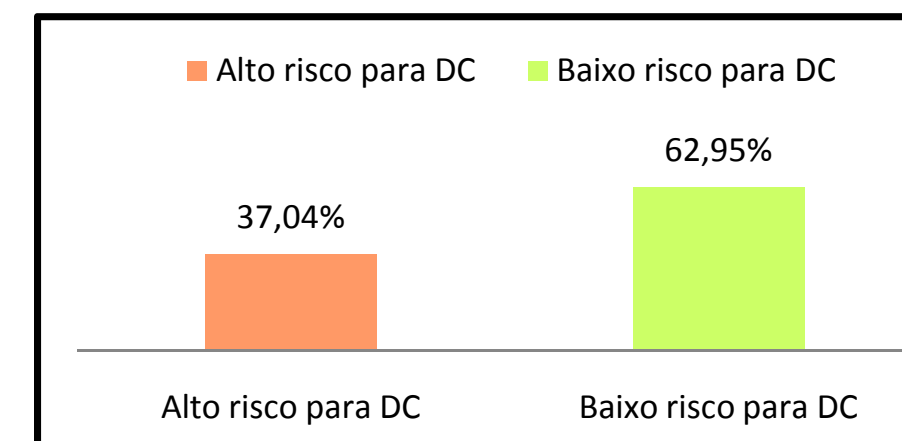

Fig. 6 - Risco de desenvolvimento de DC, de acordo com o IC, São Paulo, 2010.

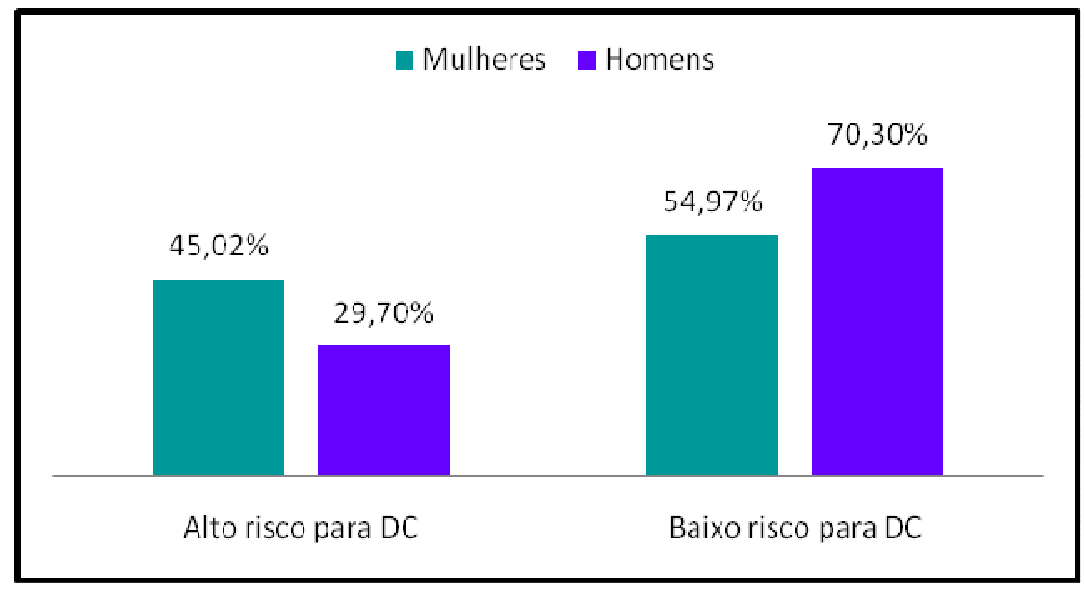

$$
\chi^{2}=17,939 ; p<0,001
$$

Fig. 7 - Distribuição percentual da amostra, segundo o sexo, para risco de desenvolvimento de doenças coronarianas, de acordo com o índice de conicidade (IC), São Paulo, 2010.

Com base nesses resultados, 0 presente estudo confirma a aparente concordância positiva do IC na determinação de risco à saúde associada ao acúmulo de gordura abdominal e risco para doenças coronarianas, entre os outros índices antropométricos. Assim como Pitanga e Lessa (2007) e Gomes (2006) que, em seus estudos, comparam indicadores antropométricos de obesidade, como o IMC e a CC em conjunto e o IC, e estes se mostraram como os melhores indicadores para o diagnóstico de risco à saúde, pois identificaram o excesso de gordura total e abdominal acima do recomendado.
O aumento da prevalência de sobrepeso e obesidade, na idade adulta, é bastante descrito na literatura; entretanto, outros estudos ainda são necessários, para identificar possíveis grupos de risco e promoção da melhora na qualidade de vida.

\section{CONCLUSÃO}

A amostra estudada apresentou uma prevalência de $26,9 \%$ de sobrepeso e $12,6 \%$ para obesidade, maior nos homens, quando comparados às mulheres. Em relação ao risco de doenças cardiovasculares segundo a circunferência de cintura e índice de 
conicidade, a maior parte $(67,7 \%)$ não apresentou risco, porém esses valores foram maiores entre as mulheres.

\section{REFERÊNCIAS BIBLIOGRÁFICAS}

ABRANTES, M. M.; LAMOUNIER, J. A.; COLONISMO, E. A. Prevalência de sobrepeso e obesidade nas regiões nordeste e sudeste do Brasil. Rev. Assoc. Med. Bras., São Paulo, v. 49, n. 2, p. 162166, 2003.

ACUÑA, K.; CRUZ, T. Avaliação do Estado Nutricional de Adultos e Idosos e Situação Nutricional da População Brasileira. Arq. Bras. Endocrinol. Metab., São Paulo, v. 48, n. 3, p. 345-361, 2004.

BATISTA FILHO, M.; RISSIN, A. A transição nutricional no Brasil: tendências regionais e temporais. Cad. Saúde Pública, Rio de Janeiro, v. 19, p. 181-S191, 2003.

BRASIL. Ministério da Saúde. Alimentação Saudável para todos: siga os dez passos. Coordenação Geral da Política de Alimentação e Nutrição, Brasília - DF, 2006.

CERVATO, A. M. et al. Dieta habitual e fatores de risco para doenças cardiovasculares. Rev. Saúde Pública, São Paulo, v. 31, n. 3, p. 227-235, 1997.

COUTINHO, D. C. et al. Condições nutricionais da população brasileira: adultos e idosos: Pesquisa Nacional sobre Saúde e Nutrição. Brasília: INAM, 1991.

FRANCISCHI, R. P. P. et al. Obesidade: atualização sobre sua etiologia, morbidade e tratamento. Rev. Nutr., Campinas, v. 13, n.1, p.17-28, jan./abr., 2000.

GOMES, M. A. Correlação entre índices antropométricos e distribuição de gordura corporal em mulheres idosas. Rev. Bras.Cine Antropom. Desempenho Hum., Florianópolis, v. 8, n. 3, p. 16-22, 2006.

LEITE, C. C. et al. Intra-abdominal thickness by ultrasonography to predict risk factors for cardiovascular disease and its correlate with anthropometric measurements. Metabolism, New York, v. 51, p. 1034-40, 2002.

LOLIO, C. A.; LATORRE, M. R. D. O. Prevalência de obesidade em localidade do Estado de São Paulo, Brasil, 1987. Rev. Saúde Pública, São Paulo, v. 23, p. 33-36, 1991.

PITANGA, F. J. G.; LESSA, I. Indicadores Antropométricos de Obesidade como instrumento de triagem para risco coronariano elevado em adultos na cidade de Salvador - Bahia. Arq. Bras. Cardiol., São Paulo, v. 85, n. 1, p. 26-31, 2005.

RANKINEN, T. et al. The prediction of abdominal visceral fat level from body composition and anthropometry: ROC analysis. Int. J. Obes. Relat. Metab. Disord., Hampshire, v. 23, p. 801-9, 1999.

SAMPAIO, L. R.; FIGUEIREDO, V. C. Correlação entre o índice de massa corporal e os indicadores antropométricos de distribuição de gordura corporal em adultos e idosos. Rev. Nutr., Campinas, v.18, n.1, p. 53-61, jan./fev., 2005.

SOUZA, L. J. de et al. Prevalência de Obesidade e Fatores de Risco Cardiovascular em Campos, Rio de Janeiro. Arq. Bras. Endocrinol. Metab., São Paulo, v. 47, n. 6, p. 669-676, dez. 2003.

VALDEZ, R. A simple model-based index of abdominal adiposity. J. Clin. Epidemiol., Oxford, v. 44, n. 9, p. 955-956, 1991. 